\title{
Investigation of Nanoparticle Agglomeration on the Effective Thermal Conductivity of a Composite Material ${ }^{*}$
}

\author{
Aaron P. Wemhoff ${ }^{1, a}$, Anthony J. Webb ${ }^{1}$ \\ ${ }^{1}$ Department of Mechanical Engineering, Villanova University, 800 Lancaster Ave., \\ Villanova, PA 19085, USA, aaron.wemhoff@villanova.edu
}

The viability of organic Phase Change Materials (PCMs), such as paraffin wax, for passive thermal management of portable electronics improves if the PCM overall bulk thermal conductivity is increased through the addition of highly conducting nanoparticles. Previous work has suggested the possibility of increasing the bulk thermal conductivity of composite materials through the controlled agglomeration of nanoparticles, yet no theoretical study has been performed to investigate the conditions under which the bulk thermal conductivity enhancement is achieved. Therefore, this study examines the influence of both spherical clustering and linear percolation network formation on the resultant bulk conductivity. This approach uses effective medium and percolation theories for unpercolated and percolated areas, respectively. Theoretical approaches are shown for spherical clustering and a 1-d conduction model of linear percolation networks, and finite element analysis (FEA) is used for a 2-d conduction model of linear percolation networks. The results for herringbone graphite nanofibers (HGNFs) in a paraffin matrix suggest that spherical clustering and linear agglomeration tend to reduce the bulk thermal conductivity, which agrees with experimental observations. However, linear percolation networks may enhance the effective thermal conductivity when large inclusion-matrix to inclusion-inclusion Kapitza resistance ratios are used.

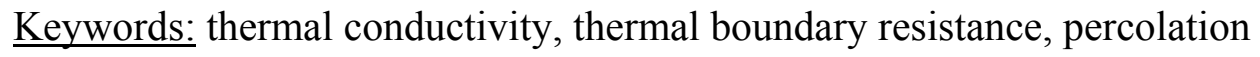

${ }^{a}$ Corresponding author; tel (610) 519-8045, fax (610) 519-7312

*Submitted for consideration for publication in the International Journal of Heat and Mass Transfer 


\section{INTRODUCTION}

The complexity for effective thermal management of portable electronics increases with the increasing demand for higher power portable electronics and smaller electronic envelopes. Although active cooling techniques, such as fans and blowers, are extremely effective, they draw valuable power from the battery. Passive cooling techniques, such as thermosyphons, heat pipes and phase change materials (PCMs), require no additional energy. Ideally, a thermal management design system should cool the components inside the electronic device while keeping the outer shell at a comfortable handling temperature. Most processors in electronics have a maximum operational temperature of $85-90^{\circ} \mathrm{C}$, but ergonomically the outer casing should be kept below $45^{\circ} \mathrm{C}$ for handheld electronics [1]. Fortunately, solid-liquid PCMs with a melting point near $50-60^{\circ} \mathrm{C}$ can not only provide passive cooling that will protect processors from overheating, but PCM use also maintains a safe handling temperature. Therefore, this study considers the use of PCMs for thermal management.

One viable PCM candidate for passively cooling portable electronics is paraffin wax. Paraffin has a latent heat near $200 \mathrm{~kJ} / \mathrm{kg}$ [2] and melt temperature in the range of $30-64^{0} \mathrm{C}$ [2], [3]. Paraffin is also non-corrosive, which is important for electronic applications. However, paraffin has a thermal conductivity of roughly $0.25 \mathrm{~W} / \mathrm{m} \mathrm{K}$ [2] at room temperature, which inhibits the energy storage capability of the PCM due to a lack of thermal diffusion. Therefore, researchers have studied the addition of carbon nanoparticles that contain a thermal conductivity possibly greater than $2,000 \mathrm{~W} / \mathrm{m} \mathrm{K}$ [4] to improve the thermal properties of PCMs [5]-[8]. Herringbone graphite nanofibers (HGNFs) are considered here as a less-expensive alternate to carbon nanotubes and graphene platelets for conductivity enhancement [5], [6], [8], [9]. When

produced with a $45^{\circ}$ crease angle, the HGNFs have a predicted cross-plane thermal conductivity 
of $27 \mathrm{~W} / \mathrm{m} \mathrm{K}$, a transverse thermal conductivity of $263 \mathrm{~W} / \mathrm{m} \mathrm{K}$ in the direction of the crease, and a transverse thermal conductivity of $1,500 \mathrm{~W} / \mathrm{m} \mathrm{K}$ normal to the crease [10].

One major issue with nano-enhanced PCMs is controlling the nanoparticle dispersion in the liquid PCM state. Nanoparticles tend to agglomerate during the solid-liquid phase transition in PCMs, especially in paraffin [5], [11], [12]. As the liquid solidifies after a single thermal cycling period, clusters of nanoparticles are observed along the grain boundaries. This agglomeration may be mitigated by introducing high density polyethylene (HDPE) [12], [13], but this approach also suppresses favorable Rayleigh-Benard convection currents. Therefore, the agglomeration of nanoparticles must be taken into consideration to be able to examine its influence on the overall thermal conductivity of a composite. Some studies show an improvement in thermal performance with the formation of percolation networks [11], [14], [15], yet others show a detrimental effect [12], so this influence is not conclusive. Therefore, this study introduces a theoretical means to predict this influence.

In order to predict the composite thermal conductivity of nano-enhanced PCMs, investigators have developed models based on approaches such as an effective medium theory (EMT) or a percolation-based theory. Traditionally, these models take into consideration the thermal boundary resistance (TBR), or Kapitza resistance, between the inclusion and the surrounding matrix [16]-[18]. However, the majority of these models focus on the influence of the inclusions on the effective thermal conductivity below the percolation threshold. An effective medium theory derived from the well-accepted model by Nan et al. [18] is commonly used to calculate the effective thermal conductivity in this study when the local volume fraction, $f$, falls below the percolation threshold, which may be defined as [19]

$$
f_{c}=\frac{\pi^{2}}{16 p}
$$


where $p=L_{i} / D$ is the inclusion aspect ratio, where $L_{i}$ and $D$ are the inclusion length and diameter, respectively. It should be noted that Eq. (1) is one of several percolation threshold models for a composite material containing uniformly distributed and oriented cylindrical or prolate inclusions, and that other models have been proposed [20]. In addition, studies by Gao and Li [21] and Wang et al. [22] have specifically observed the dependence of particle shape on the percolation threshold.

Investigators have also developed approaches, including Monte Carlo (MC) [23] and Lattice-Boltzmann [24] simulations, to predict the thermal conductivity above the percolation threshold. The MC and Lattice-Boltzmann simulations are time consuming and not as general as analytical expressions to predict the effective composite thermal conductivity. Therefore, Wemhoff [19] created a theoretical model for a composite material containing a random distribution of straight cylindrical inclusions above the percolation threshold.

Experiments [11] have indicated a deviation in the bulk composite thermal conductivity when local nanoparticle agglomeration occurs, which leads to the need to model the influence of agglomeration on bulk conductivity. Some studies, such as that by Prasher et al. [14] and Reinecke et al. [15], have successfully combined existing theories such as the Bruggeman [25] and Maxwell [26] models in order to model composites containing percolated chains of spherical particles. This study continues this line of work by, for the first time, providing parameters that directly indicate the level of agglomeration and deducing how they influence the overall bulk composite thermal conductivity, and then applying the method on a composite material with cylindrical inclusions. Furthermore, the study applies a sensitivity analysis to the results to suggest the conditions under which agglomeration may be beneficial for bulk thermal conductivity. 
This study explores three separate models of agglomeration of inclusions with the goal of establishing under which conditions agglomeration enhances or reduces the composite's bulk thermal conductivity. The first model explores spherical clustering of inclusions, whereas the remaining two models (1-d resistor network and 2-d FEA) explore the formation of linear percolation networks, keeping in mind that in reality the composite material will contain combinations of spherical clustering and linear percolation networks. These approaches will suggest the overall influence of percolation on the effective thermal conductivity of the composite material and help explain the conditions under which percolation can be beneficial towards the goal of increasing the effective thermal conductivity in a phase change material.

\section{PROCEDURE}

The cylindrical inclusion percolation method is used to determine the local thermal conductivity, $k_{e}$, when the local volume fraction of nanoparticles is above the percolation threshold. The first step to this method is determining the effective thermal conductivity of the percolated network $\left(k_{p e}\right)$ as [19]

$$
k_{p e}=\frac{k_{i}}{1+\frac{2 f}{\chi \xi(p)}}
$$

where $k_{i}$ is the inclusion thermal conductivity, $f$ is the inclusion volume fraction, $\xi(p)$ is a parameter based on inclusion geometry, and

$$
\chi=\frac{h_{c} D}{k_{i}}
$$


is an inclusion-inclusion contact Biot number, where $h_{c}$ is the inclusion-inclusion contact conductance. The geometric factor in Eq. (2) is calculated as

$$
\xi(p)=\ln \left[\frac{\sqrt{1+p^{-1}}+\sqrt{1-p^{-1}}}{\sqrt{1+p^{-1}}-\sqrt{1-p^{-1}}}\right]
$$

After determining the effective thermal conductivity of the percolated network, the Bruggeman model [25] is used to determine the effective local thermal conductivity:

$$
\left(\frac{k_{p e}}{k_{e}}\right)^{\frac{1+2 \alpha}{1-\alpha}}\left[\frac{k_{e}-k_{m}(1-\alpha)}{k_{p e}-k_{m}(1-\alpha)}\right]^{\frac{3}{1-\alpha}}=f^{3}
$$

where $k_{m}$ is the matrix thermal conductivity, and $\alpha$ is defined as

$$
\alpha=\frac{2 R_{b} k_{p e}}{D}
$$

where $R_{b}$ is the thermal boundary resistance between the inclusions and the matrix, and $D$ is the inclusion diameter. The effective local thermal conductivity is found using Eq. (5) iteratively with a standard root-finding method and a tolerance of $10^{-8}$.

If the local volume fraction is below the percolation threshold, the local thermal conductivity is determined through the use of the effective medium theory by Nan et al. [18]:

$$
k_{e}=k_{m}\left[\frac{3+f\left(2 \beta_{1}\left(1-L_{1}\right)+\beta_{3}\left(1-L_{3}\right)\right)}{3-f\left(2 \beta_{1} L_{1}+\beta_{3} L_{3}\right)}\right]
$$

where the geometrical constants of the inclusions for prolate particles are 


$$
\begin{gathered}
L_{1}=L_{2}=\frac{p^{2}}{2\left(p^{2}-1\right)}-\frac{p}{2\left(p^{2}-1\right)^{1.5}} \cosh ^{-1} p \\
L_{3}=1-2 L_{1}
\end{gathered}
$$

The parameters $\beta_{j}$ in Eq. (7) are

$$
\beta_{j}=\frac{k_{j}^{c}-k_{m}}{k_{m}+L_{j}\left(k_{j}^{c}-k_{m}\right)}
$$

where $j=\{1,2,3\}$, and

$$
k_{j}^{c}=\frac{k_{i}}{1+\frac{\gamma L_{j} k_{i}}{k_{m}}}
$$

where

$$
\gamma=\left(2+\frac{1}{p}\right)\left(\frac{2 R_{b} k_{m}}{D}\right)
$$

\subsection{Spherical Clustering}

The theoretical development for spherical clustering starts with the baseline scenario where the volume fraction of inclusions is at a uniform value $f_{0}$ throughout the entire composite material. The presence of spherical clusters may be dictated by two independent parameters. The volume fraction of clusters, $v$, represents the volume fraction of the composite material occupied by spherical clusters. The clustering density ratio, $g$, is defined as

$$
g=\frac{f_{s}}{f_{0}}
$$


where $f_{s}$ is the volume fraction of inclusions within the clusters. It follows that the volume fraction of inclusions for the region outside of the clusters is

$$
f_{n s}=f_{0} \cdot \frac{1-g v}{1-v}
$$

Therefore, one can then calculate the effective local thermal conductivity of the spherical clusters $\left(k_{s}\right)$ and the region outside of the clusters $\left(k_{n s}\right)$ using either of the approaches discussed in the previous section. Finally, the bulk conductivity is calculated using the Maxwell relation for spherical inclusions inside a matrix with no thermal boundary resistance [26],

$$
k_{b}=k_{n s} \cdot\left[1+\frac{3 v(\delta-1)}{\delta+2-v(\delta-1)}\right]
$$

where $\delta=k_{s} / k_{n s}$.

\subsection{1-d Linear Agglomeration}

This study applies agglomeration following an exponential decay of inclusion density from a straight line using the expression

$$
f(x)=\left[\frac{\beta f_{0}}{1-e^{-\beta}}\right] \exp \left(-\beta x^{*}\right)
$$

where $x^{*}=x / L_{x}$ is the dimensionless distance from the center of the agglomeration line, $L_{x}$ is the domain width, and $\beta$ is defined as the agglomeration factor:

$$
\beta=\frac{L_{x}}{L_{d}}
$$


where $L_{d}$ is the decay length associated with the inclusion distribution near the agglomeration line. An agglomeration factor of zero indicates a uniform distribution of inclusions throughout the entire domain, whereas increasing the agglomeration factor creates an increasingly denser network of inclusions along the backbone of the agglomeration line. Therefore, Eq. (16) enables agglomeration to be described by a single parameter. However, the choice of function used to describe the inclusion distribution does impact predictions of how agglomeration influences bulk thermal conductivity, as will be shown later.

The inclusion properties used in this study are shown in Table 1. The inclusion-inclusion contact conductance and inclusion-matrix thermal boundary resistance were obtained through an application of the percolated cylindrical inclusion theory to HGNF in paraffin wax [19]. The thermal conductivity tensor of HGNF was determined through molecular dynamics simulations [10], and only the axial thermal conductivity is considered in this study as the axial thermal resistance dominates over the transverse thermal resistance for HGNF. The aspect ratio of HGNF was determined from Warzoha et al. [6], and the HGNF diameter was taken from experimental measurements [27]. The overall volume fraction of inclusions was chosen to be just below the percolation threshold as determined by Eq. (1). 
TABLE 1. Inclusion-Matrix Constants Used in Simulations

\begin{tabular}{|c|c|c|}
\hline Property & Value & Basis \\
\hline $\begin{array}{c}\text { Inclusion-matrix thermal } \\
\text { boundary resistance, } R_{b}, \\
\left(\mathrm{~m}^{2} \mathrm{~K}\right) / \mathrm{W}\end{array}$ & $1 \times 10^{-10}$ & $\begin{array}{c}\text { Application of percolated cylindrical inclusion } \\
\text { theory to HGNF in Paraffin [19] }\end{array}$ \\
\hline $\begin{array}{c}\text { Inclusion-inclusion contact } \\
\text { conductance, } h_{c}, \mathrm{~W} / \mathrm{m}^{2} \mathrm{~K}\end{array}$ & $4.2 \times 10^{5}$ & $\begin{array}{c}\text { Application of percolated cylindrical inclusion } \\
\text { theory to HGNF in Paraffin [19] }\end{array}$ \\
\hline $\begin{array}{c}\text { Axial inclusion thermal } \\
\text { conductivity, } k_{i} \mathrm{~W} / \mathrm{m} \mathrm{K}\end{array}$ & 30 & Molecular dynamics simulations of HGNF [10] \\
\hline Aspect ratio, $p$ & 200 & Experimental measurement [6] \\
\hline $\begin{array}{c}\text { Inclusion Diameter, } D, \mathrm{~m} \\
\text { Overall volume fraction of } \\
\text { inclusions, } f_{0}\end{array}$ & $1 \times 10^{-7}$ & Estimate based on [27] \\
\hline $\begin{array}{c}\text { Matrix thermal conductivity, } k m, \\
\mathrm{~W} / \mathrm{m} \mathrm{K}\end{array}$ & 0.2 & Estimate based on [6] \\
\hline
\end{tabular}

The 1-d and 2-d conduction models assume an idealized case where the inclusion concentration only varies in a single direction across a domain of constant width. Figure 1 shows one of the FEA meshes used with the 2-d analysis. The figure shows that the domain width is $2 L_{x}$ by symmetry, the domain height is $L_{y}$, and the domain depth is uniform. The agglomeration line occurs at $x=0$, so Eq. (16) limits the variation of thermal properties to the $x$ direction only. The 1-d approach approximates that all conduction is in the $y$-direction only, whereas the 2-d approach contains a large domain height $\left(L_{y}=1 \mathrm{~m}\right)$ to encourage heat diffusion in the $x$-direction to obtain a more uniform temperature distribution at the top boundary. The top boundary contains a fixed heat flux of $q "=4 \mathrm{~W} / \mathrm{m}^{2}$ out of the domain, the bottom boundary contains a fixed temperature of $T_{b o t}=298 \mathrm{~K}$, and the vertical boundaries are adiabatic. 


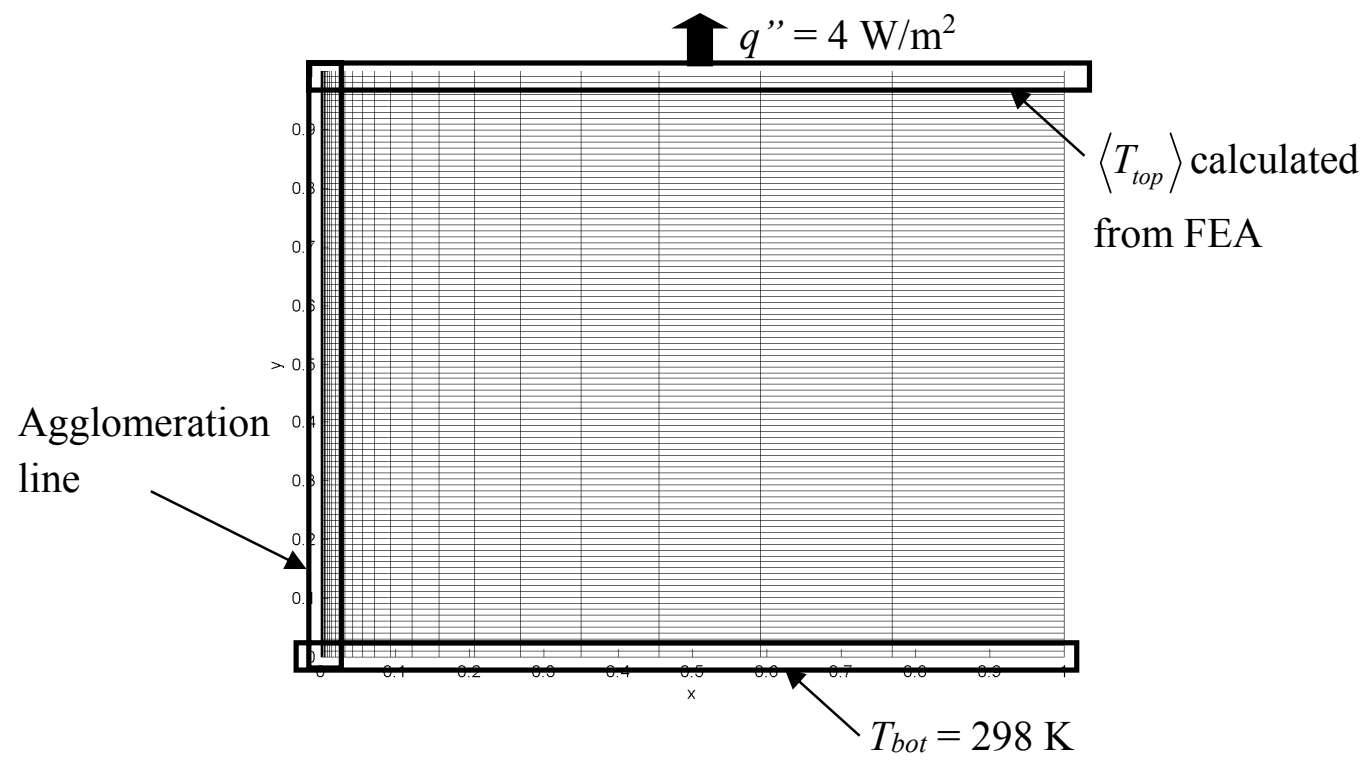

FIG. 1. Example of 2-D mesh and domain setup in order to predict influence of agglomeration on bulk thermal conductivity.

\subsubsection{1-d Conduction Model}

The 1- $\mathrm{d}$ conduction model is derived assuming heat flow is not allowed in the $x$-direction, therefore allowing for heat flow in a group of parallel, equally-sized columns of composite material. The conductance of a column $j$ is therefore

$$
G_{j}=\frac{k_{j} d x}{L_{y}}
$$

where $d x$ is the column width, and $k_{j}$ is the columnar thermal conductivity of the column. It follows that the total conductance through the domain is found as the sum of multiple parallel conductances as 


$$
G_{t o t}=\frac{d x}{L_{y}} \sum_{j} k_{j}
$$

The total conductance through the domain may be defined as $G_{t o t}=\frac{k_{b} L_{x}}{L_{y}}$, so the bulk

conductivity is calculated as

$$
k_{b}=\frac{1}{L_{x}} \sum_{j} k_{j} \cdot d x
$$

Finally, the summation can be represented as an integral since $d x$ may be assumed to be a small number:

$$
k_{b}=\frac{1}{L_{x}} \int_{0}^{L_{x}} k(x) d x
$$

Therefore, the 1-D transport assumption results in the bulk thermal conductivity equaling the averaged local thermal conductivity profile in the x-direction. Equation (21) may be integrated numerically by first applying Eq. (16) to determine the local inclusion density distribution, and then using the methods in Section 2.1 to determine the local thermal conductivity.

\subsubsection{2-d Conduction Model}

The effective thermal conductivity of each element in Fig. 1 for the 2-d conduction model is calculated using the methods in Section 2.1 by assuming an isotropic distribution of particles within each element. FEA with a $75 \times 75 \times 1$ mesh is used to obtain the steady-state temperature profile at the top boundary $\left(T_{t o p}(x)\right)$, allowing for calculation of the bulk thermal conductivity using Fourier's Law, 


$$
k_{b}=\frac{q^{\prime \prime} L_{y}}{\left(\left\langle T_{t o p}\right\rangle-T_{b o t}\right)}
$$

where $q$ " is an applied heat flux, $T_{b o t}$ is the fixed temperature at the bottom boundary, and $\left\langle T_{t o p}\right\rangle$ is the average temperature along the top boundary, defined using

$$
\left\langle T_{\text {top }}\right\rangle=\frac{1}{L_{x}} \int_{0}^{L_{x}} T_{t o p}(x) d x \approx \frac{1}{N} \sum_{i=1}^{N} T_{t o p, i}
$$

where $T_{t o p, i}$ are each of $N$ nodal temperatures along the top boundary. The standard deviation of $T_{t o p, i}$ is also determined to indicate uncertainty in $k_{b}$.

To implement this approach, a Perl script was written to modify a base input deck for the FEA conduction code Topaz3d [28]. A Topaz3d simulation provides the steady-state temperature distribution along the top boundary in Fig. 1, enabling for the calculation of the bulk thermal conductivity using Eq. (22).

The choice of mesh depends upon the applied agglomeration factor in order to validate the assumption of isotropic thermal properties within each element. A denser mesh close to the agglomeration line is required to account for larger values of nanoparticle density variation with increasing $\beta$. This increase in mesh density reduces the maximum variation in volume fraction within a single element seen in the domain,

$$
\Delta f_{e, \max }=\max \left(\Delta f_{e, j}\right)
$$

where $\Delta f_{e, j}$ is the variation of volume fraction seen in element $j$. The variation in mesh density is accomplished by altering the mesh scaling parameter 


$$
r_{m e s h}=\frac{\Delta x_{j+1}}{\Delta x_{j}}
$$

where $\Delta x_{j}$ is the width of the elements associated with a mesh column $j$. Therefore, $r_{m e s h}>1$ indicates a denser mesh towards the $x=0$ boundary. Figure 2 shows how $r_{\text {mesh }}$ influences $\Delta f_{e, \max }$. For a given value of $\beta$, if $r_{m e s h}$ is too large, then the reduction in mesh resolution away from the agglomeration line causes a corresponding increase in $\Delta f_{e}$ in elements away from the agglomeration line. If $r_{m e s h}$ is too small, then there is insufficient mesh resolution close to the agglomeration line. The optimal variation of $r_{m e s h}$ with $\beta$ is shown in Fig. 3 and is used for the predictions in this study. 


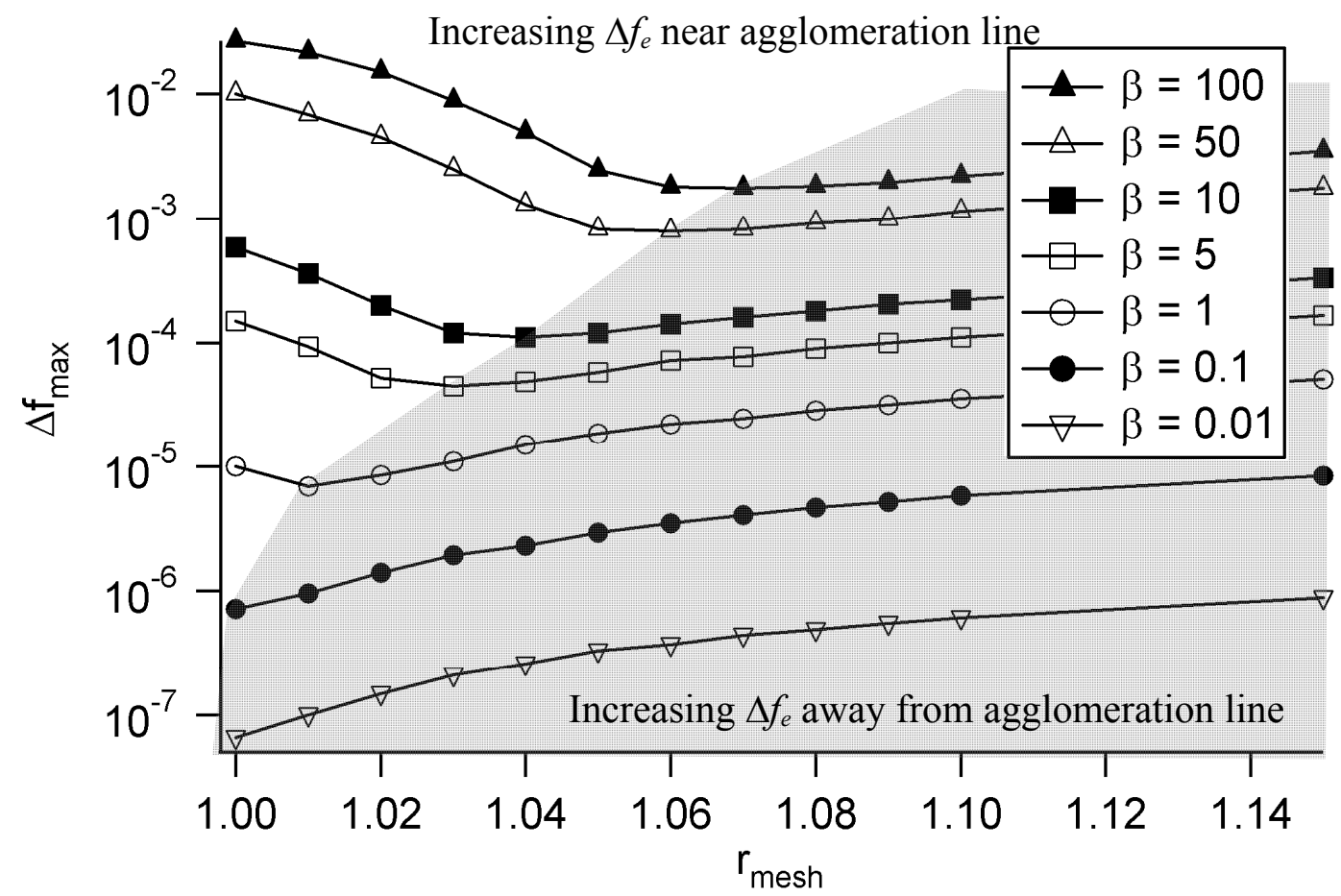

FIG 2. Influence of mesh scaling parameter on maximum observed variation in elemental inclusion volume fraction.

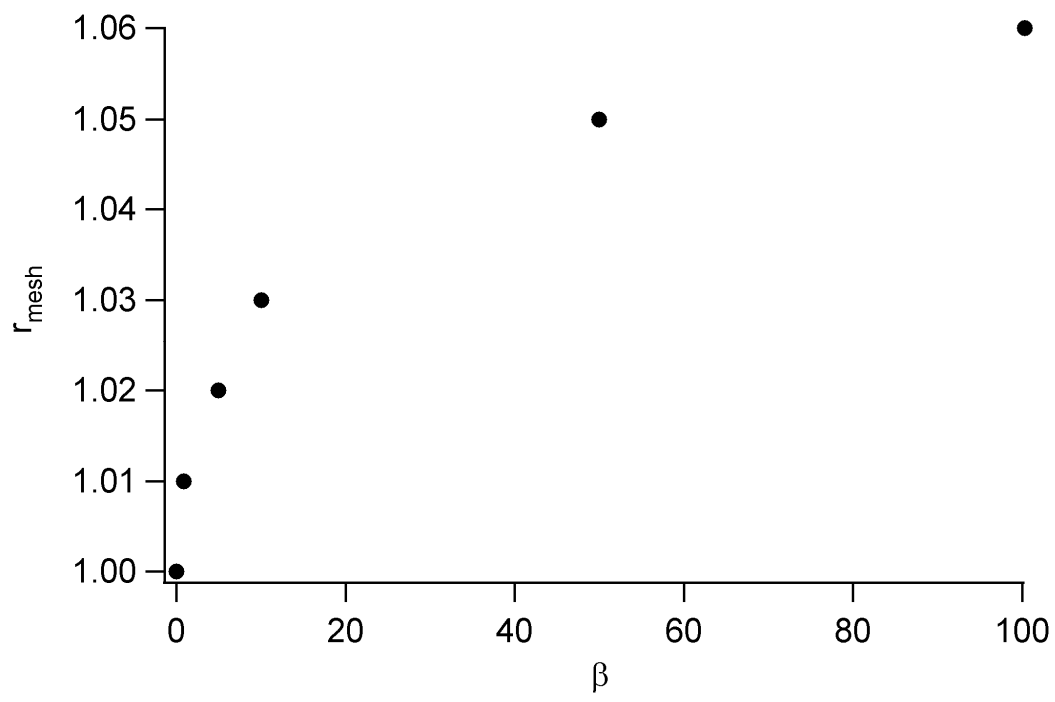

FIG. 3. Recommended mesh scaling parameter for varying percolation factors based on minimization of variation of elemental inclusion volume fraction. 


\section{RESULTS AND DISCUSSION}

\subsection{Spherical Clustering}

Figure 4 shows that increasing the amount of spherical clustering generally reduces the bulk thermal conductivity. The reason for this result, which is contrary to Maxwell's theory [26], is due to the presence of thermal boundary resistance between the particles and the background matrix. The effects of agglomeration with thermal boundary resistance causes a drop in thermal conductivity outside the clusters that is more significant than the increase in thermal conductivity inside the clusters, causing a net detrimental effect. The result is verified in that setting $g$ to 1.0 results in no change in bulk thermal conductivity since $f_{s}=f_{n s}=f_{0}$. The bulk thermal conductivity drops with increasing $g$ and $v$, suggesting that spherical clustering always reduces $k_{b}$. This can be seen mathematically under the condition that $k_{n s}$ is held constant in the tightly-packed clustering limit. In this case, Eq. (14) implies that $g$ and $v$ are coupled according to the relation

$$
v=\frac{1-F}{g-F}
$$

where $F=f_{n s} / f_{0}$ is constant. Therefore, Eq. (15) becomes

$$
k_{b}=k_{n s} \cdot\left[1+\frac{3(1-F)(\delta-1)}{(\delta+2)(g-F)-(1-F)(\delta-1)}\right]
$$

In the limit of tightly-packed clustering, $F<<1, g>>1$, and $\delta>>1$, so Eq. (27) reduces to

$$
k_{b} \approx k_{n s} \cdot\left[1+\frac{3}{g}\right]
$$

which suggests that $k_{b}$ decreases with increasing $g$ in the tightly-packed clustering limit. 


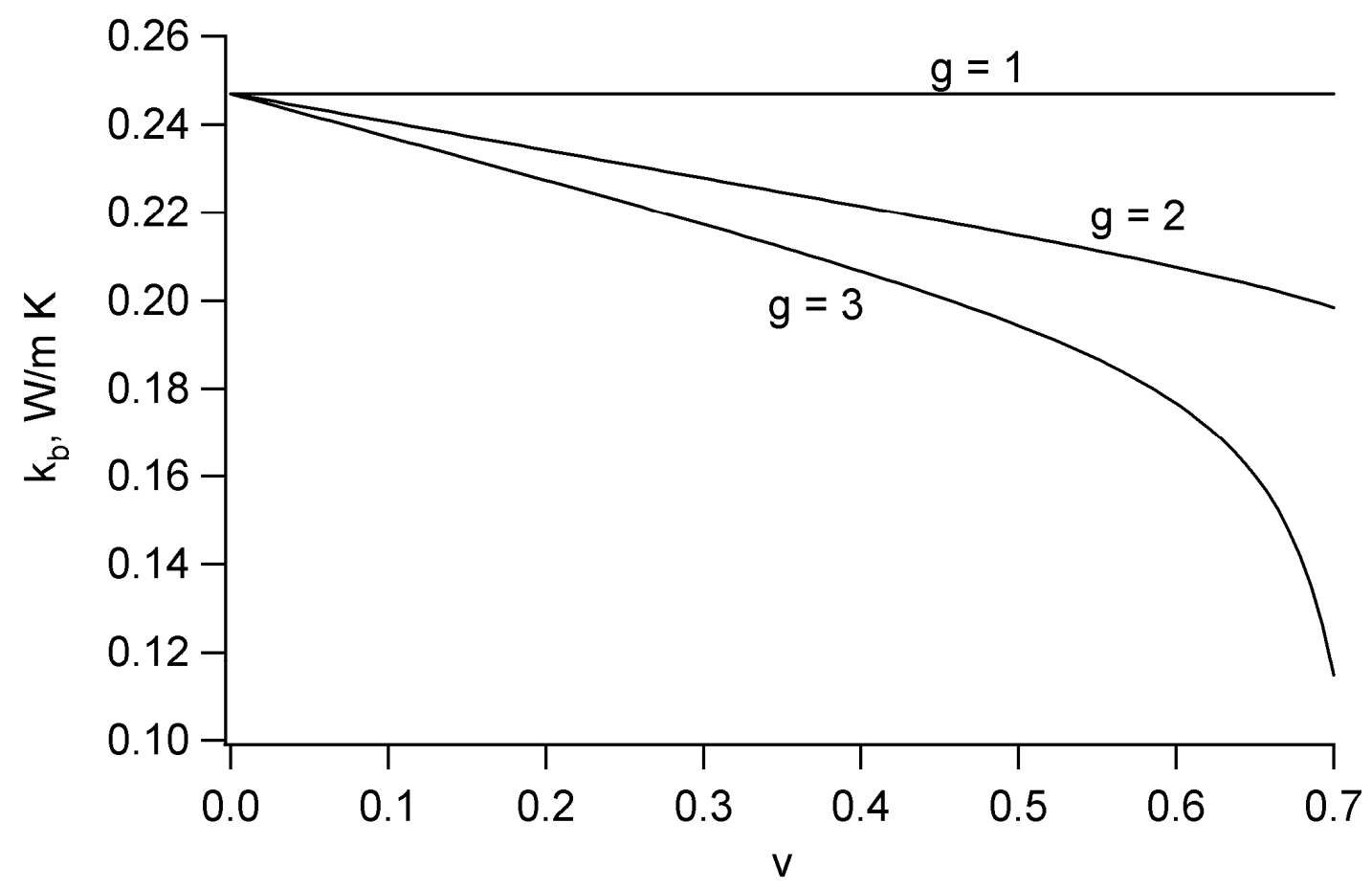

FIG. 4. Variation of bulk composite thermal conductivity with varying volume fraction of clusters and ratio of in-cluster to out-of-cluster inclusion volume fraction.

In the limit of the onset of clustering, which would occur as a perturbation of a uniform inclusion distribution, for $v=1 / 2$ an adjustment in $f_{s}$ is matched by an equivalent change in $f_{n s}$ as

$$
\begin{aligned}
& f_{s}=f_{0}\left(1+\varepsilon_{f}\right) \\
& f_{n s}=f_{0}\left(1-\varepsilon_{f}\right)
\end{aligned}
$$

It follows from Eq. (13) that $g=\left(1+\varepsilon_{f}\right)$, and Eq. (26) confirms that $v=1 / 2$. Applying differential calculus to Eq. (7) shows that the resultant variation to $k_{s}$ is equal to an opposite change in $k_{n s}$, so this change can be expressed as $\varepsilon_{k}$, allowing for the definitions 


$$
\begin{aligned}
& k_{s}=k_{b, 0}\left(1+\varepsilon_{k}\right) \\
& k_{n s}=k_{b, 0}\left(1-\varepsilon_{k}\right)
\end{aligned}
$$

where $k_{b, 0}$ is the bulk thermal conductivity in a composite with uniformly-distributed inclusions. Application of these two equations to Eq. (15) results in an expression for bulk conductivity as

$$
\frac{k_{b}}{k_{b, 0}}=\frac{\varepsilon_{k}^{2}+2 \varepsilon_{k}-3}{2 \varepsilon_{k}-3}
$$

Therefore, the change in bulk conductivity with an increase in clustering (i.e. $\varepsilon_{k}$ ), after removing higher-order terms, is

$$
\frac{1}{k_{b, 0}} \frac{d k_{b}}{d \varepsilon_{k}}=\frac{-2}{3 / \varepsilon_{k}-4}
$$

In general, $\varepsilon_{k}<<3 / 4$ since the parameter represents a small perturbation in PCM composite thermal conductivity, which is generally on the order of a few $\mathrm{W} / \mathrm{m} \mathrm{K}$. Therefore, the above derivative is less than zero, indicating that increased clustering reduces the bulk conductivity. These results suggest that spherical clustering does not increase the effective conductivity of the composite material under circumstances typical of nano-enhanced PCMs.

\subsection{Linear Agglomeration}

Figure 5 provides predictions of the variation in bulk thermal conductivity for varying percolation factors when the parameters in Table 1 are used. The figure shows that 1-d and 2-d conduction models both provide similar results. The error in 2-d calculations is approximately $0.5 \%$ for all points due to variations in nodal temperatures along the top boundary. Mesh independence for the $2-\mathrm{d}$ results was shown by running a test case of $\beta=100$ with a $50 \times 50 \times 1$ 
mesh, which indicated a difference in $k_{b}$ of $0.15 \%$ compared to the $75 \times 75 \times 1$ mesh used to predict the data in the figure.

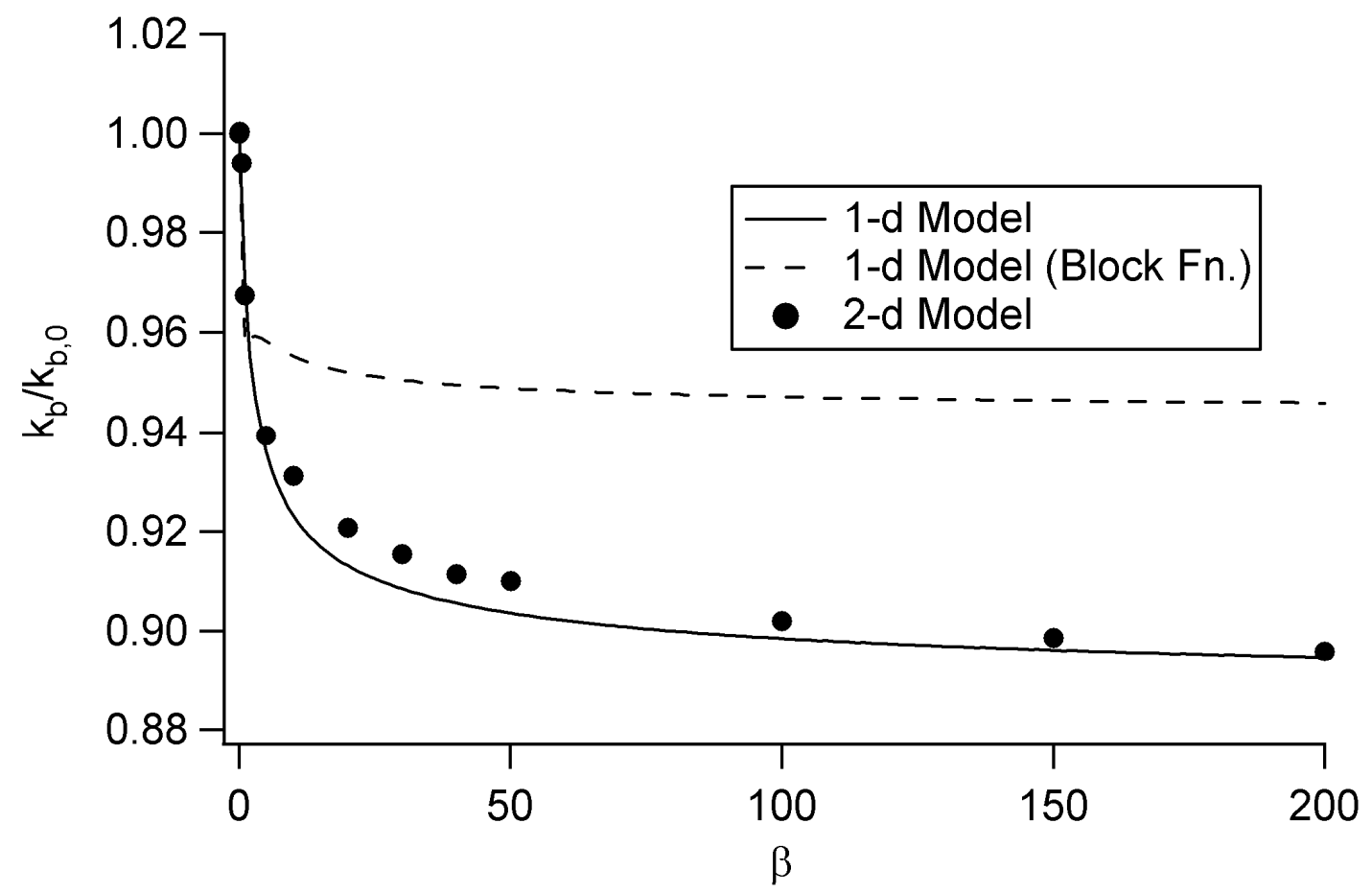

FIG. 5. Variation of bulk thermal conductivity for various percolation factors and prediction methods.

The figure shows the dropoff in bulk thermal conductivity with increasing linear agglomeration of inclusions. This result is consistent with an observed reduction in thermal transport for paraffin-HGNF mixtures seen in experiments [12]. One can see that the curve asymptotically trends to 0.885 , which is equal to the ratio $\mathrm{km} / \mathrm{kb}, 0$, indicating that the impact of the conduction network in the agglomerated region becomes ineffective due to its narrow width. 
It should be noted that the choice of function used to define agglomeration impacts the predicted influence of agglomeration on the effective bulk thermal conductivity. A block function was defined for comparison to Eq. (16) such that half of the inclusions in both Eq. (16) and the block function are located on either side of the position $x_{1}^{*}$ as shown in Fig. 6. The 1-d predictions using the block function are provided in Figure 5, which shows that the functions deviate significantly when agglomeration is present (i.e. $\beta>>1$ ), primarily due to the nonzero presence of inclusions in the block function far from the agglomeration line in this limit.

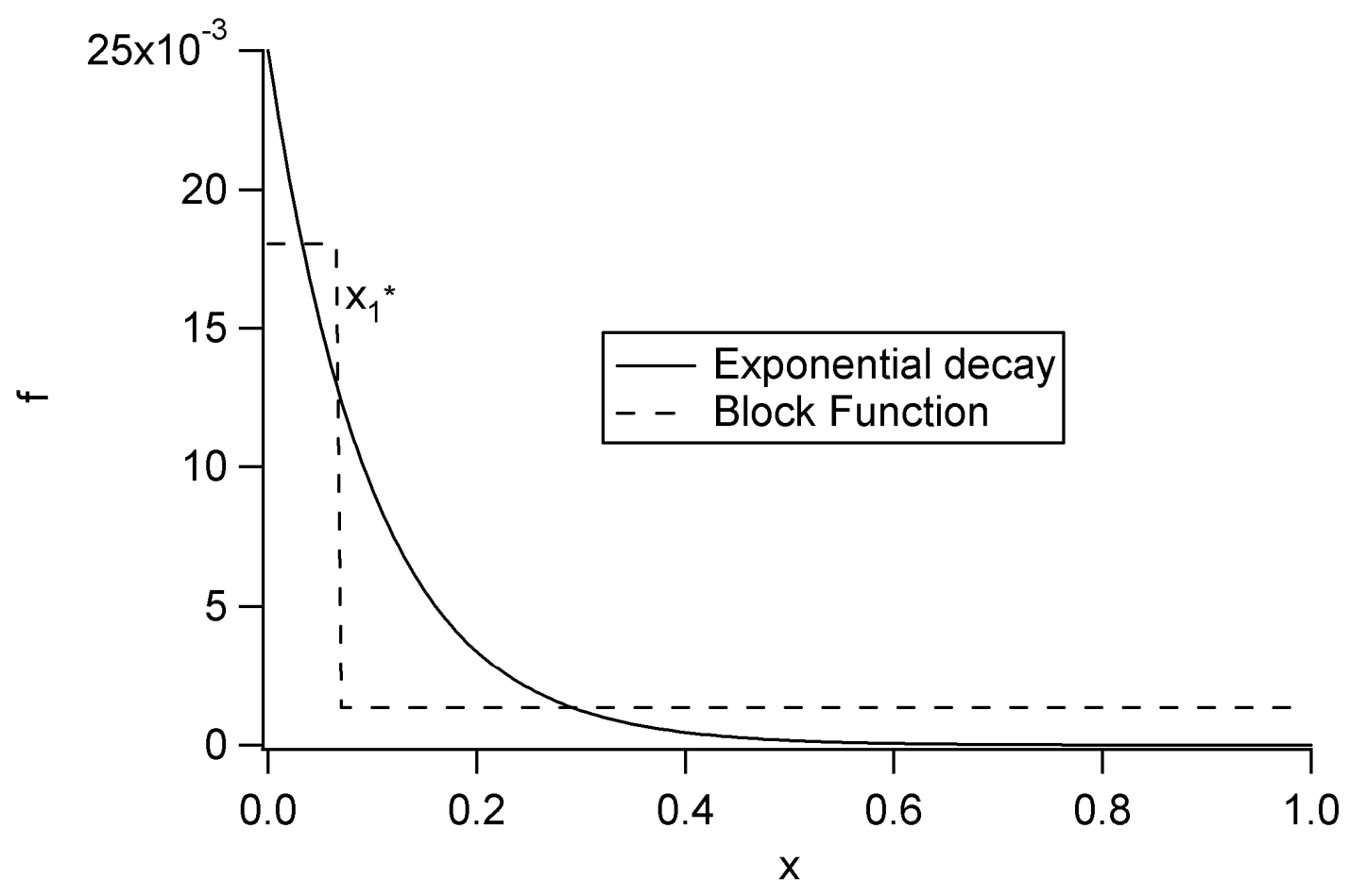

FIG. 6. Volume fraction profiles for $\beta=10$ and the parameters in Table 1.

A screening-type sensitivity analysis was also performed to provide an indication of the circumstances by which linear agglomeration can increase the bulk thermal conductivity. This analysis was performed using the 2-d conduction model with $\beta=100$ and by varying $D, L, k_{i}, R_{b}$, 
and $h_{c}$ by $+/-50 \%$ from their values in Table 1 . These variables may be grouped into dimensionless parameters according to the equation

$$
\frac{k_{b}}{k_{b, 0}}=f\left(p, \frac{k_{i}}{k_{m}}, R_{b} h_{c}\right)
$$

Figure 7 shows that in general the dominant factor for increasing $k_{b} / k_{b, 0}$ is the product $R_{b} h_{c}$, which is essentially the ratio of inclusion-matrix to inclusion-inclusion Kaptiza resistances. Increasing $R_{b} h_{c}$ effectively reduces the relative thermal resistance through the percolation network compared to the unpercolated regions of the domain.

The results from Fig. 7 suggest that composite materials with large $R_{b} h_{c}$ may enable $k_{b} / k_{b, 0}>1$. To test this hypothesis, a simulation of single-walled carbon nanotubes (SWCNTs) in a PMMA matrix $\left(k_{m}=0.18 \mathrm{~W} / \mathrm{m} \mathrm{K}\right)$ was performed. The parameter values used in the model are $D=14 \mathrm{~nm}, p=26, k_{i}=287 \mathrm{~W} / \mathrm{m} \mathrm{K}, R_{b}=9 \times 10^{-8} \mathrm{~m}^{2} \mathrm{~K} / \mathrm{W}$, and $h_{c}=5.7 \times 10^{7} \mathrm{~W} / \mathrm{m}^{2} \mathrm{~K}$ consistent with the application of the percolated cylindrical inclusions method [19] to experimental data by Guthy et al. [29]. The overall volume fraction of SWCNTs is kept at 0.0025 . The corresponding value of $R_{b} h_{c}$ is 5.13, which is much larger than the values for HGNF in PCM. Therefore, the dimensionless parameters are more favorable towards enhancing bulk thermal conductivity compared to HGNF in paraffin wax. The resultant calculated bulk thermal conductivity for SWCNTs in PMMA with $\beta=100$ is $k_{b} / k_{b, 0}=1.04+/-0.02$, which therefore shows a benefit from the presence of the linear agglomeration network. 

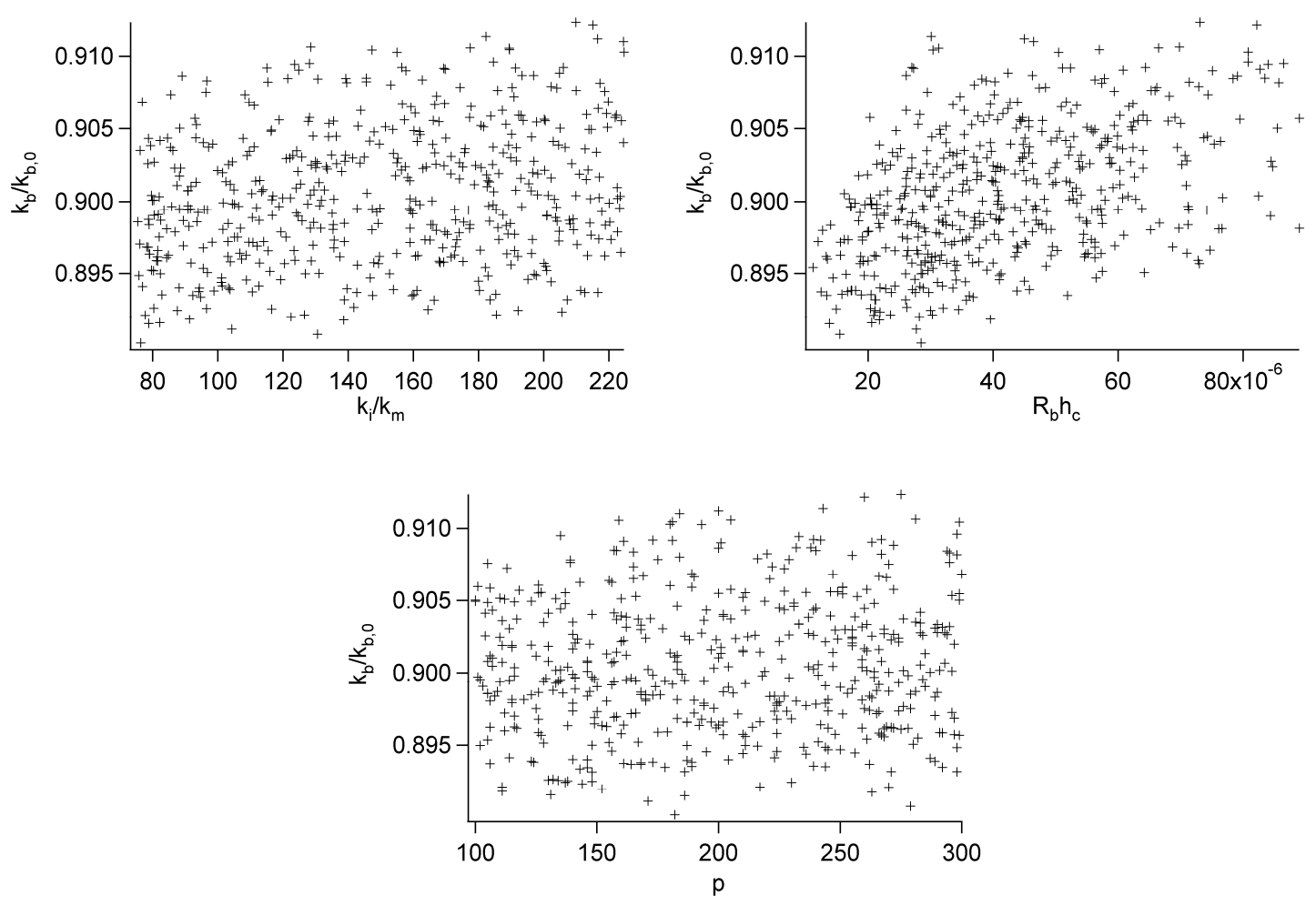

FIG. 7. Variation in predicted bulk thermal conductivity for various dimensionless parameters.

\section{CONCLUSIONS}

This study suggests that agglomeration of nanoparticles in the form of spherical clusters always reduces the effective thermal conductivity of a nano-enhanced composite material. The predictions in this study also provide an explanation as to why studies have shown instances of linear agglomeration networks enhancing the bulk thermal conductivity, whereas in other studies the presence of linear agglomeration networks decreases the bulk thermal conductivity. Work still needs to be done to determine how to effectively tune the inclusion-inclusion and inclusionmatrix Kapitza resistances in order to achieve large values of $R_{b} h_{c}$ that favor thermal conductivity enhancement via linear agglomeration formation. Future work also requires 
experimental method development to ensure linear agglomeration network formation while avoiding spherical cluster formation.

\section{ACKNOWLEDGEMENTS}

This material is based upon work supported by the National Science Foundation under Grant No. CBET-0931507. Any opinions, findings, and conclusions or recommendations expressed in this material are those of the author(s) and do not necessarily reflect the views of the National Science Foundation. 


\section{NOMENCLATURE}

$D$

$f$

$f_{c}$

$f_{n s}$

$f_{s}$

$f_{0}$

F

$g$

G

$h_{c}$

$k_{b}$

$k_{b, 0}$

$k_{e}$

$k_{i}$

$k_{m}$

$k_{n s}$
Inclusion diameter, $\mathrm{m}$

Inclusion volume fraction, dimensionless

Percolation threshold, dimensionless

Inclusion volume fraction outside clusters, dimensionless

Inclusion volume fraction within clusters, dimensionless

Overall inclusion volume fraction, dimensionless

Ratio of volume fraction of inclusions outside clusters to the overall volume fraction of inclusions, dimensionless

Clustering density ratio, dimensionless

Conductance, $\mathrm{W} / \mathrm{K}$

Inclusion-inclusion contact conductance, $\mathrm{W} / \mathrm{m}^{2} \mathrm{~K}$

Effective bulk thermal conductivity, W/m K

Effective bulk thermal conductivity in a composite with uniformly distributed inclusions, $\mathrm{W} / \mathrm{m} \mathrm{K}$

Effective local thermal conductivity, W/m K

Inclusion thermal conductivity, $\mathrm{W} / \mathrm{m} \mathrm{K}$

Matrix thermal conductivity, W/m K

Effective thermal conductivity outside clusters, W/m K 


\begin{tabular}{|c|c|}
\hline$k_{p e}$ & Effective percolation backbone thermal conductivity, W/m K \\
\hline$k_{1}^{c}, k_{2}^{c}, k_{3}^{c}$ & Adjusted inclusion conductivities used in effective medium model, $\mathrm{W} / \mathrm{m} \mathrm{K}$ \\
\hline$k_{s}$ & Effective thermal conductivity within clusters, $\mathrm{W} / \mathrm{m} \mathrm{K}$ \\
\hline$L_{d}$ & Decay length in inclusion distribution near the boundary, $\mathrm{m}$ \\
\hline$L_{x}$ & Domain width, $\mathrm{m}$ \\
\hline$L_{i}$ & Inclusion length, $\mathrm{m}$ \\
\hline$L_{y}$ & Domain height, $\mathrm{m}$ \\
\hline$L_{1}, L_{2}, L_{3}$ & Geometric factors in effective medium model, dimensionless \\
\hline$N$ & Number of nodal temperatures along the top boundary, dimensionless \\
\hline$p$ & Inclusion aspect ratio, dimensionless \\
\hline$q "$ & Heat flux, $\mathrm{W} / \mathrm{m}^{2}$ \\
\hline$r_{\text {mesh }}$ & Mesh scaling parameter, dimensionless \\
\hline$R_{b}$ & Inclusion-matrix thermal boundary resistance, $\mathrm{m}^{2} \mathrm{~K} / \mathrm{W}$ \\
\hline$T_{b o t}$ & Temperature at bottom of domain, $\mathrm{K}$ \\
\hline$T_{\text {top }}$ & Temperature at top of domain, $\mathrm{K}$ \\
\hline$v$ & Volume fraction of clusters, dimensionless \\
\hline$\alpha$ & Parameter used in Bruggeman model, dimensionless \\
\hline
\end{tabular}


Percolation factor, dimensionless

$\beta_{1}, \beta_{2}, \beta_{3}, \gamma \quad$ Parameters used in effective medium model, dimensionless

$\delta$

Ratio of thermal conductivity inside clusters versus outside clusters, dimensionless

$\varepsilon$

Small change in volume fraction or thermal conductivity ratio, dimensionless

$\Delta f_{e}$

Variation of elemental inclusion volume fraction, dimensionless

$\xi$

Geometric factor in percolated cylindrical inclusions method

$\chi$

Inclusion-inclusion contact Biot number, dimensionless 


\section{REFERENCES}

[1] M. K. Berhe, "Ergonomic temperature limits for handheld electronic devices," presented at the ASME InterPACK, 2007, pp. 1041-1046.

[2] I. Dincer and M. A. Rosen, Thermal Energy Storage: Systems and Applications. Wiley, 2010.

[3] N. Ukrainczyk, S. Kurajica, and J. Šipušić, "Thermophysical comparison of five commercial paraffin waxes as latent heat storage materials," Chem. Biochem. Eng. Q., vol. 24, no. 2, pp. 129-137, 2010.

[4] A. A. Balandin, "Thermal properties of graphene and nanostructured carbon materials," Nat. Mater., vol. 10, no. 8, pp. 569-581, Aug. 2011.

[5] R. D. Weinstein, T. C. Kopec, A. S. Fleischer, E. D’Addio, and C. A. Bessel, "The Experimental exploration of embedding phase change materials with graphite nanofibers for the thermal management of electronics," J. Heat Transf., vol. 130, p. 042405, 2008.

[6] R. J. Warzoha, R. M. Weigand, and A. S. Fleischer, "Temperature-dependent thermal properties of a paraffin phase change material embedded with herringbone style graphite nanofibers," Appl. Energy, 2014.

[7] R. J. Warzoha and A. S. Fleischer, "Effect of graphene layer thickness and mechanical compliance on interfacial heat flow and thermal conduction in solid-liquid phase change materials," ACS Appl. Mater. Interfaces, 2014.

[8] K. Chintakrinda, R. Weinstein, and A. S. Fleischer, "Quantification of the Impact of Embedded Graphite Nanofibers on the Transient Thermal Response of Paraffin Phase Change Material Exposed to High Heat Fluxes," J. Heat Transf., vol. 134, p. 071901, 2012.

[9] K. Chintakrinda, R. Weinstein, and A. S. Feischer, "A Direct Comparison of Three Different Material Enhancement Methods on the Transient Thermal Response of Paraffin Phase Change Material Exposed to High Heat Fluxes," Int. J. Therm. Sci., vol. 50, no. 9, pp. 16391647, 2011.

[10] M. H. Khadem and A. P. Wemhoff, "Thermal conductivity predictions of herringbone graphite nanofibers using molecular dynamics simulations," J. Chem. Phys., vol. 138, p. $084708,2013$.

[11] R. T. Zheng, J. W. Gao, J. J. Wang, and G. Chen, "Reversible temperature regulation of electrical and thermal conductivity using liquid-solid phase transitions," Nat. Commun., vol. 2, Apr. 2011.

[12] R. Ehid, R. D. Weinstein, and A. S. Fleischer, "The Shape Stabilization of Paraffin Phase Change Material to Reduce Graphite Nanofiber Settling During the Phase Change Process,"

Energy Convers. Manag., vol. 57, pp. 60-67, 2012.

[13] R. Ehid and A. S. Fleischer, "Development and Characterization of Paraffin-Based Shape Stabilized Energy Storage Materials," Energy Convers. Manag., vol. 53, pp. 84-91, 2012.

[14] R. Prasher, W. Evans, P. Meakin, J. Fish, P. Phelan, and P. Keblinski, "Effect of aggregation on thermal conduction in colloidal nanofluids," Appl. Phys. Lett., vol. 89, no. 14, p. 143119 , Oct. 2006.

[15] B. N. Reinecke, J. W. Shan, K. K. Suabedissen, and A. S. Cherkasova, "On the anisotropic thermal conductivity of magnetorheological suspensions," J. Appl. Phys., vol. 104, no. 2, p. 023507, Jul. 2008.

[16] L. Gao, X. F. Zhou, and Y. L. Ding, "Effective thermal and electrical conductivity of carbon nanotube composites," Chem. Phys. Lett., vol. 434, no. 4-6, pp. 297-300, Feb. 2007. 
[17] S. H. Xie, Y. Y. Liu, and J. Y. Li, "Comparison of the effective conductivity between composites reinforced by graphene nanosheets and carbon nanotubes," Appl. Phys. Lett., vol. 92, no. 24, p. 243121, Jun. 2008.

[18] C. W. Nan, R. Birringer, D. R. Clarke, and H. Gleiter, "Effective thermal conductivity of particulate composites with interfacial thermal resistance," J. Appl. Phys., vol. 81, no. 10, pp. 6692-6699, May 1997.

[19] A. P. Wemhoff, "Thermal conductivity predictions of composites containing percolated networks of uniform cylindrical inclusions," vol. 62, pp. 255-262, 2013.

[20] Y. Pan, G. J. Weng, S. A. Meguid, W. S. Bao, Z. H. Zhu, and A. M. S. Hamouda, "Percolation threshold and electrical conductivity of a two-phase composite containing randomly oriented ellipsoidal inclusions," J. Appl. Phys., vol. 110, no. 12, p. 123715, Dec. 2011.

[21] L. Gao and Z. Li, "Effective medium approximation for two-component nonlinear composites with shape distribution," J. Phys. Condens. Matter, vol. 15, no. 25, p. 4397, 2003.

[22] Y. Wang, G. J. Weng, S. A. Meguid, and A. M. Hamouda, "A continuum model with a percolation threshold and tunneling-assisted interfacial conductivity for carbon nanotubebased nanocomposites," J. Appl. Phys., vol. 115, no. 19, p. 193706, May 2014.

[23] S. Kumar, M. A. Alam, and J. Y. Murthy, "Effect of percolation on thermal transport in nanotube composites," Appl. Phys. Lett., vol. 90, no. 10, p. 104105, Mar. 2007.

[24] M. Wang, Q. J. Kang, and N. Pan, "Thermal conductivity enhancement of carbon fiber composites," Appl. Therm. Eng., vol. 29, no. 2-3, pp. 418-421, Feb. 2009.

[25] D. A. G. Bruggeman, "Berechnung Verschiedener Physikalischer Konstanten von Heterogenen Substanzen. I. Dielektrizitatskonstanten und Leitfahigkeiten der Mischkorper aus Isotropen Substanzen," Аnпu. Phys. Leipz., vol. 24, pp. 636-679, 1935.

[26] J. C. Maxwell, A Treatise on Electricity and Magnetism. Cambridge, UK: Oxford University Press, 1904.

[27] C. A. Bessel, K. Laubernds, N. M. Rodriguez, and R. T. K. Baker, "Graphite nanofibers as an electrode for fuel cell applications," J. Phys. Chem. B, vol. 105, no. 6, pp. 1115-1118, 2001.

[28] A. P. Wemhoff, M. A. Havstad, and A. B. Shapiro, "TOPAZ3D Users Manual - A 3dimensional Finite Element Heat Transfer Code," Lawrence Livermore National Laboratory, Livermore, CA, 2007.

[29] C. Guthy, F. Du, S. Brand, K. I. Winey, and J. E. Fischer, "Thermal conductivity of single-walled carbon nanotube/PMMA nanocomposites," J. Heat Transf.-Trans. Asme, vol. 129, no. 8, pp. 1096-1099, Aug. 2007. 